\title{
Comparison on Efficacy and Safety of Three Inpatient Insulin Regimens for Management of Non-Critical Patients with Type 2 Diabetes
}

\author{
Eman Said $^{1 *}$, Samar Farid ${ }^{1}$, Nirmeen Sabry ${ }^{1}$, May Fawzi ${ }^{2}$ \\ ${ }^{1}$ Department of Clinical Pharmacy, Faculty of Pharmacy, Cairo University, Cairo, Egypt; ${ }^{2}$ Departments of Internal Medicine, Faculty \\ of Medicine, Cairo University, Cairo, Egypt. \\ Email:"gaviota_3011@hotmail.com
}

Received July $18^{\text {th }}, 2013$; revised August $25^{\text {th }}, 2013$; accepted September $15^{\text {th }}, 2013$

Copyright (C) 2013 Eman Said et al. This is an open access article distributed under the Creative Commons Attribution License, which permits unrestricted use, distribution, and reproduction in any medium, provided the original work is properly cited.

\begin{abstract}
Background: Hyperglycemia in hospitalized patients is associated with poor clinical outcomes. Scheduled Subcutaneous Insulin therapy has been recommended for better glycemic control. Aims: To compare efficacy and safety of traditional sliding scale insulin (SSI) versus modified 70/30 insulin versus basal plus supplemental scale (SS) insulin regimens for glycemic control of inpatients with diabetes. Methods: In a prospective trial, 62 patients with diabetes were randomized to receive either hospital SSI $(\mathrm{N}=22)$, or twice daily 70/30 insulin plus supplemental lunchtime insulin for $\mathrm{BG} \geq 150 \mathrm{mg} / \mathrm{dL}(\mathrm{N}=21)$ or once every night glargine plus prandial glulisine for $\mathrm{BG} \geq 150 \mathrm{mg} / \mathrm{dL}(\mathrm{N}=19) .70 / 30$ insulin and glargine were started respectively at 0.4 and $0.2 \mathrm{U} / \mathrm{kg} / \mathrm{day}$ for $\mathrm{BG} \leq 200 \mathrm{mg} / \mathrm{dL}$ or 0.5 and $0.3 \mathrm{U} / \mathrm{kg} / \mathrm{day}$ for BG above $200 \mathrm{mg} / \mathrm{dL}$. Results: Starting at BG level of $204 \pm 68,200 \pm 50$ and $241 \pm 94 \mathrm{mg} / \mathrm{dL}$ in SSI, 70/30 insulin and glargine/glulisine groups respectively, $(\mathrm{F}(2,35.47)=1.467, \mathrm{p}=0.244$, Welch test $)$, mean daily BG after first day of hospitalization was statistically significant $(\mathrm{F}(2,35.58)=7.043, \mathrm{p}=0.003$, Welch test) lower in $70 / 30$ insulin group $(171 \pm 38 \mathrm{mg} / \mathrm{dL})$ compared to $(218 \pm 71 \mathrm{mg} / \mathrm{dL})$ in SSI group $(\mathrm{p}=0.026)$ and $(225 \pm 65 \mathrm{mg} / \mathrm{dL})$ in glargine/glulisine group $(\mathrm{p}=0.01)$. Conclusions: With poorly educated nursing staff, basal plus SS insulin failed to provide adequate glycemic control. However, tailored 70/30 insulin regimen resulted in statistically significant glycemic control compared to traditional SSI.
\end{abstract}

Keywords: Type 2 DM; Sliding Scale Insulin; 70/30 Insulin; Basal Insulin; Supplemental Insulin

\section{Introduction}

Diabetes Mellitus (DM) is a major, emerging clinical and public health problem in Egypt and worldwide. Based on 1985 WHO diagnostic criteria for diabetes and the studies being conducted in Egypt in 1995 [1], the prevalence of DM adjusted to world population was $11.4 \%$ in 2010 and estimated to be $13.7 \%$ in 2030 [2]. By the year 2025 , it is estimated that $13.3 \%$ of the population 20 years of age will have diabetes [3].

Patients with DM have a threefold greater chance of hospitalization compared to those without DM [4]. The management of DM in hospitals is generally considered secondary in importance when compared to the condition that prompted admission [5-7]. Despite the increasing body of evidence to support the tight glycemic control in

${ }^{*}$ Corresponding author. hospitalized patients, blood glucose control continues to be deficient and is frequently overlooked in general medicine and surgery services [5].

Traditional sliding scale insulin (SSI) as sole regimen is no more recommended $[8,9]$. This reactive approach is not based on physiologic principles of glucose regulation [10], where it treats hyperglycemia after it has already occurred instead of preventing its occurrence, leading in turn to rapid changes in blood glucose levels, exacerbating both hyperglycemia and hypoglycemia, and can lead to iatrogenic diabetic ketoacidosis (DKA) [7,11].

Insulin 70/30 is a combination product consisting of $30 \%$ regular insulin and 70\% isophane NPH insulin. The soluble component, when injected 30 minutes before a meal, aims to lower postprandial glucose excursions, while NPH provides basal insulin coverage for both breakfast and dinner. Together, they should provide in- 
sulin coverage for existing hyperglycemia, as well as any subsequent hyperglycemia [12].

The combination of a long-acting basal insulin such as glargine, or detemir with a rapid-acting prandial insulin such as aspart, lispro or glulisine has been recommended as a more physiological approach to glucose control in the hospital than SSI [7,13-15] .

Although Scheduled Subcutaneous Insulin therapy (SCI) has been recommended for better glycemic control in hospitals [16], SSI regimens continue to be used by many clinicians in the Egyptian's hospitals and this often results in undesirable outcomes. A better controlling tool for DM is clearly needed.

Accordingly, the aim of this study was to compare the efficacy and safety of traditional SSI to modified tailored 70/30 insulin to basal plus SS insulin regimen for glycemic control in hospitalized patients with type $2 \mathrm{DM}$.

\section{Methods}

\subsection{Patients}

63 non-surgical patients with a known history of type 2 DM for longer than 3 months admitted to general medicine wards were recruited. Further inclusion criteria included, age between 18 - 64 year old, treatment with diet alone, any combination of oral antidiabetic agents and/or insulin before admission. Exclusion criteria included subjects with hyperglycemia without any known history of DM, presence of diabetic ketoacidosis (DKA), patients admitted to intensive care unit (ICU), subjects expected to undergo surgery during the hospitalization course, patients with clinically relevant hepatic disease, impaired renal function (serum creatinine $\geq 3.0 \mathrm{mg} / \mathrm{dL}$ ), systemic infections or pregnancy. Also, patients on medications known to interfere with the blood glucose level (either increasing or decreasing) were excluded from the study.

All patients were recruited from the department of internal medicine, Cairo University teaching hospitals, Cairo, Egypt. All patients were screened for their demographic and baseline clinical characteristics before conducting the study.

\subsection{Study Design}

This was a prospective, open-label parallel, randomized study in which all oral anti-diabetic agents were discontinued on admission and selected patients were randomly allocated to receive one of three regimens: either traditional hospital sliding scale insulin (SSI) protocol or to receive 70/30 insulin $\left(\right.$ Mixtard $^{\circledR}$ 70/30) regimen twice daily plus supplemental regular insulin at lunch time or to receive once every night (glargine (lantus ${ }^{\circledR}$ ) plus three times supplemental glulisine (apidra ${ }^{\circledR}$ ) before each meal.
Insulin management was directed by the hospital specific designed protocol and was carried out daily by members of the internal medicine residency-training program. Glycosylated hemoglobin (HbAlC) was measured on the first day of hospitalization. The primary admission illnesses and preadmission DM treatments have been recorded. No follow-up visit after discharge was included in the study design.

Modified basal/bolus regimen and split-mixed insulin protocols adopted in Umpierrez et al., studies [14,17] were applied to patients treated with glargine/glulisine and 70/30 insulin respectively as detailed in Table 1. For SSI group, regular insulin was administered three times daily subcutaneously approximately $30 \mathrm{~min}$ before meal for $\mathrm{BG}>150 \mathrm{mg} / \mathrm{dL}$ (or every 8 hours if a patient was not eating) according to Table 2.

The results of BG values were presented as pre-meal glucose and mean daily BG during the hospital stay from the day of admission. The primary outcome of the study was to determine differences in glycemic control between treatment groups as measured by mean daily BG concentration during their hospital stay. Secondary outcomes included number of hypoglycemic events, number of episodes of severe hyperglycemia, length of hospital stay (LOS), and mortality rate. Hypoglycemic episodes were classified as major (BG $\leq 40 \mathrm{mg} / \mathrm{dL}$ or associated with impaired mental status or loss of consciousness), or minor (BG between 40 and $59 \mathrm{mg} / \mathrm{dL}$ ) events. Hyperglycemic events were defined as BG $>300$ $\mathrm{mg} / \mathrm{dL}$.

\subsection{Statistical Analysis}

Statistical analysis was performed using the SPSS software package version 16. Statistical significance was defined as $p<0.05$. For continuous variables, one way analysis of variance (ANOVA) followed by Tukey-Post hoc or Welch test followed by Games Howell-Post hoc was performed, while the Chi square test or Fisher's exact test was used for qualitative variables.

\section{Results}

A total of 63 patients with Type 2 DM admitted to general medicine services were recruited. In this study, the patients were randomly assigned to receive either hospital SSI $(\mathrm{N}=22)$, or $70 / 30$ insulin $(\mathrm{N}=21)$ or glargine/glulisine insulin $(\mathrm{N}=20)$. One patient in the glargine/glulisine group was excluded due to the development of DKA. There were no significant differences in demographic and clinical characteristics of study patients on admission (Table 3). The most common admitting illnesses included a variety of cerebrovascular (45.2\%), cardiovascular $(11.3 \%)$, gastroin-testinal $(8.1 \%)$, pul- 
Table 1. Modified Insulin Regimens applied to subjects treated with glargine/gluisine and Premixed 70/30 insulin.

1. Glargine plus supplemental glulisine regimen

\subsection{Basal insulin: glargine}

1. Patients treated with diet or oral agents before admission:

- Hold oral antidiabetic drugs on admission.

- Starting glargine insulin dose:

$0.2 \mathrm{U} / \mathrm{kg} /$ day for admission $\mathrm{BG} \leq 200 \mathrm{mg} / \mathrm{dL}$

$0.3 \mathrm{U} / \mathrm{kg} / \mathrm{day}$ for BG exceeding $200 \mathrm{mg} / \mathrm{dL}$

2. Patients treated with insulin before admission:

- Glargine/glulisine treated patients should be started at half amount of the total outpatient insulin dose as glargine.

- Premixed 70/30 insulin treated patients should be started at half amount of the total outpatient insulin dose as glargine.

- Conversion unit glargine-per-unit 70/30 insulin.

- In both cases: give glargine once daily in the evening around 8:00 pm.

1.2. Supplemental insulin: glulisine

- Give glulisine three times before each meal for $\mathrm{BG} \geq 150 \mathrm{mg} / \mathrm{dL}$ according to scale provided in Table 2 .

Hold glulisine if a patient is not able to eat to prevent hypoglycemia.

2. Premixed 70/30 insulin plus supplemental lunchtime regular insulin regimen

\subsection{Premixed 70/30 insulin}

1. Patients treated with diet or oral agents before admission:

- Hold oral antidiabetic drugs on admission.

- Starting Premixed 70/30 insulin dose:

$0.2 \mathrm{U} / \mathrm{kg} / \mathrm{day}$ for admission $\mathrm{BG} \leq 200 \mathrm{mg} / \mathrm{dL}$

$0.4 \mathrm{U} / \mathrm{kg} /$ day for BG exceeding $200 \mathrm{mg} / \mathrm{dL}$

2. Patients treated with insulin before admission:

- Premixed 70/30 insulin treated patients should be started at the same outpatient insulin dose and schedule.

- In both cases: give two thirds of total daily dose (TDD) before breakfast and 1/3 of TDD before dinner.

2.2. Supplemental insulin: regular insulin

- Give regular insulin before lunch for $\mathrm{BG} \geq 150 \mathrm{mg} / \mathrm{dL}$ according to scale provided in Table 2 .

Hold regular insulin if a patient is not able to eat to prevent hypoglycemia.

\section{Insulin adjustment}

- If fasting and premeal blood glucose is $<150 \mathrm{mg} / \mathrm{dl}$ (in the absence of hypoglycemia): no change

- If fasting and premeal blood glucose is between 150 and $180 \mathrm{mg} / \mathrm{dl}$ (in the absence of hypoglycemia): increase the TDD of 70/30 insulin or glargine by $10 \%$ every day.

- If fasting and premeal blood glucose is $>180 \mathrm{mg} / \mathrm{dl}$ (in the absence of hypoglycemia): increase the TDD of 70/30 insulin or glargine dose by $20 \%$

- If a patient develops hypoglycemia $(<60 \mathrm{mg} / \mathrm{dl})$ : decrease the TDD of $70 / 30$ insulin or glargine insulin dose by $20 \%$.

- The distribution of 70/30 insulin doses followed the 2/3 AM and 1/3 PM scheme.

\section{Blood glucose monitoring}

Blood glucose will be measured before each meal (i.e. before breakfast, before lunch and before dinner) or every 8 hours if the patient was not eating using the same hospital point of care glucose meter.

In addition, blood glucose will be measured at any time if a patient experiences symptoms of hypoglycemia or if requested by the treating physician.

Table 2. Insulin Dosing Scale based on BG levels as being used by Kasr El-Aini Teaching Hospitals, Cairo, Egypt.

\begin{tabular}{cccccccc}
\hline BG (mg/dL) & $<150$ & $151-200$ & $201-250$ & $251-300$ & $301-350$ & $351-400$ & $>400^{*}$ \\
\hline Insulin (IU) & No & 5 & 10 & 15 & 20 & 25 & 30 \\
\hline
\end{tabular}

"Call the internal medicine resident, BG: Blood Glucose.

monary (4.8\%), infectious (3.2\%), and other disorders $(27.4 \%)$. There was no significant difference among reasons leads to hospital admission between the three treated groups $(\mathrm{p}=0.163)$.

\subsection{Efficacy (Primary) Outcomes}

Starting at BG level prior to treatment of $204 \pm 68 \mathrm{mg} / \mathrm{dL}$ in SSI, $200 \pm 50 \mathrm{mg} / \mathrm{dL}$ in 70/30 insulin and $241 \pm 94$ 
Management of Non-Critical Patients with Type 2 Diabetes

Table 3. Characteristics of study subjects on admission.

\begin{tabular}{|c|c|c|c|c|}
\hline Parameter & SSI protocol $\mathrm{N}=22$ & $\begin{array}{c}70 / 30 \text { insulin regimen } \\
\mathrm{N}=21\end{array}$ & $\begin{array}{l}\text { Glargine/glulisine insulin } \\
\text { regimen } \mathrm{N}=19\end{array}$ & $\mathrm{P}^{*}$ \\
\hline Age $(y r) \pm S D$ & $55 \pm 7$ & $55 \pm 7$ & $54 \pm 11$ & $\mathrm{NS}^{\#}$ \\
\hline Range (years) & $37-64$ & $45-64$ & $23-64$ & \\
\hline Gender (No. of males (\%)) & $7(31.8 \%)$ & $9(42.9 \%)$ & $5(26.3 \%)$ & $\mathrm{NS}^{*}$ \\
\hline Body weight $(\mathrm{kg}) \pm \mathrm{SD}$ & $73 \pm 12$ & $78 \pm 20$ & $75 \pm 11$ & $\mathrm{NS}^{\#}$ \\
\hline Range (Kg) & $45-95$ & $46-126$ & $50-102 \mathrm{~kg}$ & \\
\hline Body mass index $\left(\mathrm{kg} / \mathrm{m}^{2}\right) \pm \mathrm{SD}$ & $31 \pm 5$ & $31 \pm 7$ & $31 \pm 6$ & $\mathrm{NS}^{\#}$ \\
\hline Range $\left(\mathrm{kg} / \mathrm{m}^{2}\right)$ & $17-41$ & $17-47$ & $25-42.5$ & \\
\hline \multicolumn{5}{|c|}{ Diabetes treatment prior to admission, $\mathrm{N}(\%)$} \\
\hline Insulin & $12(54.5 \%)$ & $11(52.4 \%)$ & $12(63.2 \%)$ & $\mathrm{NS}^{¥}$ \\
\hline Insulin plus oral antidiabetic agents & $0(0 \%)$ & $1(4.8 \%)$ & $1(5.3 \%)$ & $N S^{\ddagger}$ \\
\hline Oral antidiabetic agent as monotherapy & $8(36.4 \%)$ & $8(38.1 \%)$ & $3(15.8 \%)$ & $\mathrm{NS}^{\ddagger}$ \\
\hline Multiple oral antidiabetic agents & $2(9.1 \%)$ & $0(0 \%)$ & $3(15.8 \%)$ & $\mathrm{NS}^{*}$ \\
\hline No pharmacological agent & $0(0 \%)$ & $1(4.8 \%)$ & $0(0 \%)$ & $N S^{*}$ \\
\hline $\mathrm{BG}$ on $\operatorname{admission}(\mathrm{mg} / \mathrm{dL}) \pm \mathrm{SD}$ & $253 \pm 68$ & $240 \pm 49$ & $262 \pm 90$ & $\mathrm{NS}^{\$}$ \\
\hline Range (mg/dL) & $131-440$ & $159-330$ & $121-410$ & \\
\hline $\mathrm{BG}$ prior treatment $(\mathrm{mg} / \mathrm{dL}) \pm \mathrm{SD}$ & $204 \pm 68$ & $200 \pm 50$ & $241 \pm 94$ & $\mathrm{NS}_{\$}$ \\
\hline Range (mg/dL) & $92-336$ & $129-352$ & $108-410$ & \\
\hline Hemoglobin $\mathrm{A} 1 \mathrm{C}(\%) \pm \mathrm{SD}$ & $8.8 \pm 2.6$ & $9.4 \pm 2.3$ & $9.1 \pm 3.0$ & $\mathrm{NS}_{\#}$ \\
\hline Range \% & $5.5-13.3$ & $5.4-13.4$ & $4.6-14.3$ & \\
\hline Creatinine $(\mathrm{mg} / \mathrm{dL}) \pm \mathrm{SD}$ & $1.2 \pm 0.60$ & $0.98 \pm 0.35$ & $1.19 \pm 0.61$ & $\mathrm{NS}^{\$}$ \\
\hline Range (mg/dL) & $0.60-2.50$ & $0.57-2.10$ & $0.64-2.86$ & \\
\hline \multicolumn{5}{|l|}{ Admitting illnesses, N (\%) } \\
\hline Cerebrovascular & $10(45.5 \%)$ & $12(57.1 \%)$ & $6(31.6 \%)$ & $\mathrm{NS}^{*}$ \\
\hline Cardiovascular & $4(18.2 \%)$ & $1(4.8 \%)$ & $2(10.5 \%)$ & $\mathrm{NS}^{¥}$ \\
\hline Gastrointestinal & $3(13.6 \%)$ & $1(4.8 \%)$ & $1(5.3 \%)$ & $\mathrm{NS}_{¥}$ \\
\hline Pulmonary & $0(0 \%)$ & $2(9.5 \%)$ & $1(5.3 \%)$ & $\mathrm{NS}^{\ddagger}$ \\
\hline Infectious & $0(0 \%)$ & $2(9.5 \%)$ & $0(0 \%)$ & $\mathrm{NS}^{\ddagger}$ \\
\hline Others & $5(22.7 \%)$ & $3(14.3 \%)$ & $9(52.9 \%)$ & $\mathrm{NS}^{¥}$ \\
\hline
\end{tabular}

Data are presented as mean $\pm \mathrm{SD}, \mathrm{SD}$ : Standard Deviation, ${ }^{*}$ Level of significance at $\mathrm{p}<0.05$, NS: Non-Significant, ${ }^{\#}$ Analysis of Variance (ANOVA) test at $\mathrm{p}<$ $0.05,{ }^{\$}$ Welch test at $\mathrm{p}<0.05,{ }^{\sharp}$ Fisher's Exact test at $\mathrm{p}<0.05$, BG: Blood Glucose, Normal value of Creatinine is $0.6-1.3 \mathrm{mg} / \mathrm{dL}$.

$\mathrm{mg} / \mathrm{dL}$ in glargine/glulisine $(\mathrm{F}(2,35.47)=1.467 \mathrm{p}=$ $0.244)$, Welch $\mathrm{F}$ test). There was a statistically significant difference in glycemic control after day 1 of hospitalization between treated groups $(\mathrm{F}(2,35.58)=7.043, \mathrm{p}=$ 0.003 , Welch F test). The mean daily BG level after the first day of hospitalization was significantly lower in the $70 / 30$ insulin group $(171 \pm 38 \mathrm{mg} / \mathrm{dL})$ compared to $218 \pm$ $71 \mathrm{mg} / \mathrm{dL}$ in the SSI group $(\mathrm{p}=0.026)$ and $225 \pm 65$ $\mathrm{mg} / \mathrm{dL}$ in the glargine/glulisine group $(\mathrm{p}=0.01)$. There was no statistically significantly difference between the SSI group and glargine/glulisine group $(p=0.947)$. BG level of patients in SSI group continued to rise through their hospital stay while there was fluctuation in glargine/ glulisine group (Figure 1).

Although 70/30 insulin group showed lower mean morning BG level $(180 \pm 52 \mathrm{mg} / \mathrm{dL})$ versus $(194 \pm 76$ and $205 \pm 69 \mathrm{mg} / \mathrm{dL}$ ) in glargine/glulisine group and SSI group respectively. There was no statistically significant 


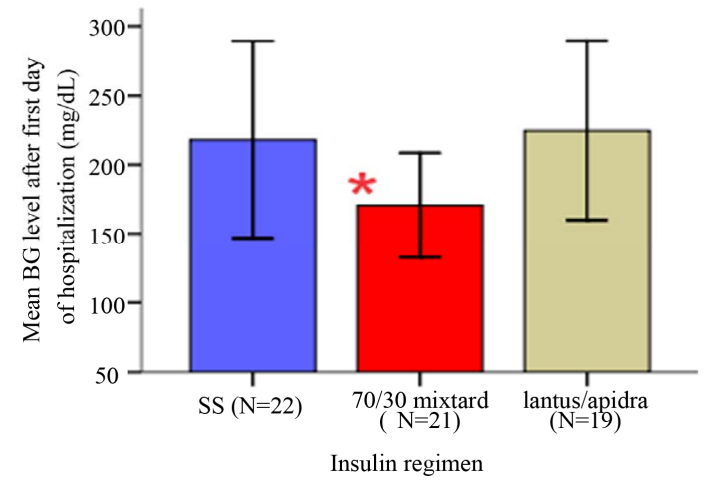

Figure 1. The Relationship between different type of insulin regimens and BG level after first day of hospitalization (Welch, ${ }^{*}$ Significant, $p=\mathbf{0 . 0 0 3}$ ).

difference in mean morning BG level between treated groups $(\mathrm{F}(2,59)=0.772, \mathrm{p}=0.46)$.

A significantly $(\mathrm{F}(2,59)=3.7, \mathrm{p}=0.031)$ lower mean total daily BG level during entire hospital stay of $179 \pm$ $36 \mathrm{mg} / \mathrm{dL}$ was observed in 70/30 insulin compared to 222 $\pm 67 \mathrm{mg} / \mathrm{dL}$ in the SSI group $(\mathrm{p}=0.049)$.

To ensure glycemic control before discharge, the mean BG levels were compared during the last day of hospitalization between treated groups $(\mathrm{F}(2,59)=3.69, \mathrm{p}=$ 0.031 ). A significantly lower BG level of $168 \pm 38$ $\mathrm{mg} / \mathrm{dL}$ was observed in 70/30 insulin group compared to $220 \pm 76 \mathrm{mg} / \mathrm{dL}$ in SSI group $(\mathrm{p}=0.025)$.

\subsection{Safety (Secondary) Outcomes}

There was a statistically significant difference in the mean length of stay (LOS) between treated groups (F $(2,59)=4.7, p=0.013)$. LOS was statistically significantly shorter ( $4 \pm 1.6$ day) in patients treated with 70/30 insulin compared to $(6 \pm 2.5$ day) those received the SSI therapy $(p=0.011)$. There was neither statistically significant difference in LOS between 70/30 insulin and glargine/glulisine group $(5.4 \pm 2.5$ day, $\mathrm{p}=0.11)$ nor between SSI group and glargine/glulisine group $(\mathrm{p}=0.66)$ (Figure 2).

Although about one fourth (10 \pm 7 IU) of total daily (glargine/glulisine) doses were missed, a significantly ( $\mathrm{F}$ $(2,59)=7.82, \mathrm{p}=0.01)$ higher dose was administrated in subjects of the glargine/glulisine group $(45 \pm 18 \mathrm{IU})$ compared to that administrated ( $26 \pm 14 \mathrm{IU})$ in SSI group $(p=0.001)$. There was neither statistically significant difference in mean total insulin dose between 70/30 insulin and glargine/glulisine group $(34 \pm 13 \mathrm{IU}, \mathrm{p}=$ $0.073)$ nor between $70 / 30$ insulin and SSI group $(\mathrm{p}=$ $0.201)$.

About $80 \%$ of total daily missed (glargine/glulisine) was attributed to missed glargine dose which represented $8 \pm 7$ IU while daily glulisine missed doses $1 \pm 2$ IU.
This resulted in Unequal distribution between glargine and glulisine dose where, the mean daily glulisine dose was ( $28 \pm 16 \mathrm{IU})$ represented approximately $2 / 3$ of total glargine/glulisine dose while the mean daily glargine dose was only $17 \pm 9 \mathrm{IU}$ (Figure 3). The mean total daily missed insulin doses were $(2 \pm 2)$ and $(2 \pm 3)$ IU in SSI and 70/30 insulin respectively. Fifteen patients (79\%) of (glargine/glulisine) group missed at least one glargine dose during their hospital stay.

There were no episodes of hypoglycemia recorded in the 70/30 insulin group. Only a single patient in SSI group experienced eight hypoglycemic events (BG between 40 and $59 \mathrm{mg} / \mathrm{dL}$ ). In glargine/glulisine, two cases experienced major hypoglycemic episode of $\mathrm{BG} \leq 40 \mathrm{mg} / \mathrm{dL}$. One of them experience two episodes hypoglcemia while the other had only one episode. Three patients experienced hypoglycemic events of BG be- tween 40 and 59 $\mathrm{mg} / \mathrm{dL}$ and were not associated with adverse outcomes. Hyperglycemia defined as BG $>300 \mathrm{mg} / \mathrm{dL}$, occurred at least one time in 15 patients (68.2\%) in SSI insulin group versus 10 patients $(52.6 \%)$ in glargine/glulisine group and four patients $(19 \%)$ in $70 / 30$ insulin group $(\mathrm{p}=$ 0.005). No deaths were reported in any of the three treated. Summary of highlighted finding results (Table 4)

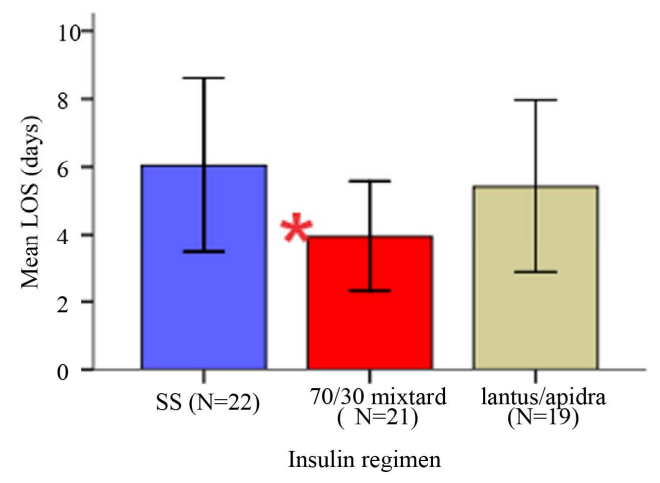

Figure 2. The relationship between different type of insulin regimens and LOS (ANOVA, "Significant, $p=0.013$ ).

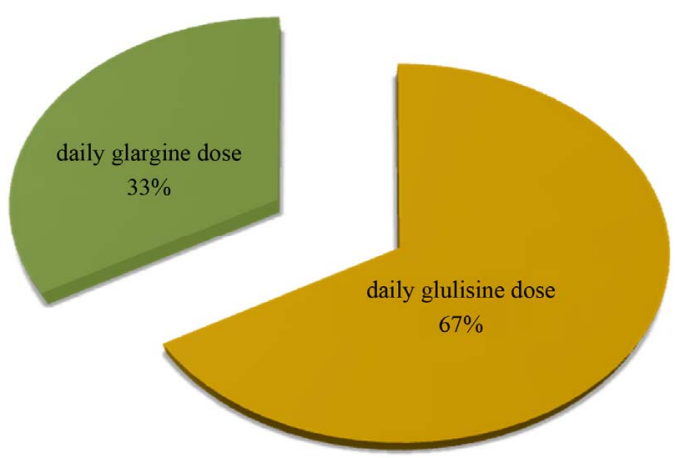

Figure 3. Distribution of glargine and glulisine in total daily (glargine/glulisine) dose. 
Management of Non-Critical Patients with Type 2 Diabetes

Table 4. Effect of Different insulin regimens on LOS, Total insulin units and Mean BG levels measured at various time.

\begin{tabular}{ccccc}
\hline Parameter & SSI N $=22$ & $70 / 30$ insulin N $=21$ & Glargine/glulisine N=19 & Level of significance \\
\hline BG after Day1 (mg/dL) & $218 \pm 71$ & $171 \pm 38$ & $225 \pm 65$ & $0.003^{*}$ \\
LOS (days) & $6 \pm 2.57$ & $4 \pm 1.62$ & $5.42 \pm 2.54$ & $0.013^{\#}$ \\
Mean total BG (mg/dL) & $222 \pm 67$ & $179 \pm 36$ & $221 \pm 67$ & $0.031^{\#}$ \\
BG last day (mg/dL) & $220 \pm 76$ & $168 \pm 38$ & $202 \pm 70$ & $0.031^{\#}$ \\
Morning BG (mg/dL) & $205 \pm 69$ & $180 \pm 52$ & $194 \pm 76$ & $0.467^{\#}$ \\
Mean Total insulin units (IU) & $26 \pm 14$ & $34 \pm 14$ & $45 \pm 18$ & $0.001^{\#}$ \\
\hline
\end{tabular}

Data are presented as mean \pm SD, SD: Standard Deviation, ${ }^{*}$ Welch test, LOS: Length of Stay, ${ }^{*}$ ANOVA test at level of significance $<0.05$, BG: Blood Glucose.

\section{Discussion}

Evidence from observational and interventional studies indicated that, hyperglycemia occurring during hospitalization (HDH) in critical and noncritical illness was associated with an increased risk of complications and mortality $[5,7,18,19]$.

In the present study, glulisine doses were given before each meal according to sliding scale based on premeal BG level rather than three fixed doses with additional supplemental doses as shown in Umpierrez et al., study [14]. This modification gave the investigators great flexibility on selecting the most appropriate gluisine doses from first time without the need for dose correction and thought to minimize confusion among nursing staff. Although SSI doses illustrated in the present study seem to be somehow aggressive scale, which has not been sufficiently tested in the literature, it represents a commonly used sliding scale in both governmental and private hospitals in Egypt. Although the glycemic target in majority of non-critical care setting is to maintain premeal BG level below $140 \mathrm{mg} / \mathrm{dL}$ [9], this aggressive scale provides no prandial coverage when $\mathrm{BG}$ values are under $150 \mathrm{mg} / \mathrm{dl}$. This is acceptable with respect to the risk of hypoglycemia [9]

Modified tailored 70/30 insulin regimens including twice-daily premixed insulin plus supplemental lunch insulin resulted in a significant improvement in glycemic control throughout the hospital stay. Starting at a similar BG concentration, the mean BG level after the first day of hospital stay was significantly lower in the 70/30 insulin group compared to the other regimens. This finding supports the better glycemic control reported in Schoeffler et al., pilot study utilizing 70/30 insulin versus SSI [20] and goes along with Liebl et al., [21] who showed that, twice daily injections of premixed insulin were comparable to intensive insulin treatment in insulin naive subjects. Glycemic control with glargine/glulisine was comparable with SSI which opposes Umpierrez et al., finding [14] who reported better glycemic control achiev- ed with basal/bolus insulin than with SSI alone. Our finding opposes recommendations on utilizing SCI as a more physiological approach to control blood glucose in hospitals [16].

Because of the inadequate duration of action of NPH insulin and the undesirable peak activity at $4-6$ hour after injection [20,22], some Type 2 DM patients on twice daily treatment with premixed insulin, may complain of hypoglycemia before lunch, necessitating a reduction in the insulin dose at breakfast. However, reducing the insulin dosage at breakfast may cause an increase in evening plasma glucose levels; indicating the limitation of this insulin regimen. Moreover; in Egypt, consuming homemade meals provided by caregivers is a common hospital break regulation which may result in increase in both lunch and evening BG levels. To overcome limitation with in twice daily NPH regimen, biphasic insulin analogues have been given three times a day and shown to be as efficacious as basal-bolus regimen [23], but accompanied with more minor hypoglycemic episodes compared to biphasic human insulin twice daily, but this was not statistically significant [24]. The safety of thrice daily biphasic human insulin regimen is still not well established [25]. An alternative option was to add midday prandial insulin to biphasic insulin based regimen as shown by Holman et al. [26]. This regimen was applied in the present study through introducing supplemental insulin dose at lunch time when $\mathrm{BG}>150 \mathrm{mg} / \mathrm{dL}$ resulted in better glycemic control. Our adopted protocol used a similar concept to that offered by Yamada et al., [27] who suggested additing glinide, a novel rapid-acting insulin secretagogue, once a day at lunch-time to twice daily injections of premixed insulin.

The continuous elevation in BG level in SSI group supports the medical fact that the use of SSI as sole treatment is ineffective and associated with several problems [28-31]. SSI is a reactive response to hyperglycemia $[7,32]$ and is based on illogical assumption that insulin sensitivity is the same for all patients [20]. 
In the present study, despite a proper administration schedule and monitoring design, poor glycemic control was observed in the glargine/glulisine insulin group, which may be attributed to nursing's errors. With low education and poorly trained nursing staff, giving two different types of insulin at night created a great source of confusion and resulted in some cases, in nurse selfdecision of giving only one type of insulin. If the missed insulin was glargine, this resulted in great BG fluctuation overall the next day. This problem was confirmed by interviewing the patients rather than interviewing the nurses, who would not admit it and most times confirmed by reviewing patient's medication sheet, where no glargine doses were recorded to be administrated. This was a very difficult point to control by the investigators who normally were available during the morning doses only. Although no studies in Egypt have focused on quality of nursing staff, there was a great agreement among practicing physicians and residents on both shortage and poor education level of practicing nursing staff in Cairo University teaching hospitals where the study was conducted. Unfortunately, same problem could be easily extrapolated in other governmental hospitals in Egypt. Different research studies concluded that, hospital working conditions and the adequacy of nurse staffing are important predictors of variation in hospital patient outcomes [33-38].

In the non-critical care setting under investigation in this study, patients remained on the same SSI regimen despite widely varying insulin requirements resulting in undesirable hypoglycemic and hyperglycemic events that were observed in more than $68 \%$ of patients treated with SSI. Hyperglycemia (BG $>300 \mathrm{mg} / \mathrm{dL}$ ) observed in more than half of the patients treated with glargine/glulisine in addition to higher BG morning which may also be attributed to missing glargine night dose as previously mentioned.

$70 / 30$ insulin is a combination product containing both short- and intermediate-acting insulins. Pharmacodynamic profile of 70/30 insulin (30 minutes onset, peak after 4 - 8 hours, effect, and duration up to 24 hours) [20] provides better basal insulin coverage, resulted in lower mean BG morning in 70/30 group compared to the SSI group and fewer subsequent hyperglycemic events. These results indicate that, the 70/30 insulin regimen provides better glycemic control compared to SSI hospital protocol which goes along with Schoeffler et al., pilot study utilizing 70/30 insulin regimen [20].

The presence of hyperglycemia is associated with prolonged hospital stay, disability after hospital discharge, and death [5]. The mean hospital LOS was significantly shorter in patients treated with 70/30 insulin compared to those in the SSI group. This finding opposed the one re- ported by Schoeffler et al. [20], which showed no significant differences in mean hospital LOS comparing $70 / 30$ insulin regimen versus SSI. This may be explained by the significant glycemic control observed in patients treated with 70/30 insulin compared to SSI, and thus faster recovery in patient's condition confirmed by significant lower BG reading on last day of hospitalization. However, LOS was comparable in glargine/glulisine group and SSI group, which, agrees with the finding reported by Umpierrez et al., study [14].

Patients treated with glargine/glulisine group received approximately two times higher total daily insulin dose than those treated with SSI. This higher dose could not explain hypoglycemic events occured in glargine/glulisine group since in Umpierrez et al., [14] despite higher approximately triple insulin dose, the use of the basal /bolus insulin regimen was safe and was associated with a low rate of hypoglycemic events. However, in the present study, and surprisingly, there was an unequal distribution between glargine and glulisine in the total administrated dose where glulisine contributed a great proportion in total daily dose. As a result of missing glargine night dose, higher glulisine doses were administrated to correct hyperglycemia. Hyperglycemia over correction resulted in subsequent hypoglycemia. The risk of hypoglycemia increased particularly in that subject group due to inappropriate timing of rapid acting insulin in relation to meals. The practice of giving insulin just before the meal created a second source of error among nursing staff who almost forgot to ask patients on basal/bolus regimen to take their meal immediately after bolus insulin injection instead of waiting 30 minutes as they used to do with their home insulin. The average total daily insulin dose was slightly higher in the 70/30 insulin group compared to SSI; however, this was expected as shown in Schoeffler et al., pilot study [20].

Despite the evidence supporting inpatient glycemic control and guidelines recommending tighter inpatient glycemic control, clinical practice in Egyptian hospitals is slow to change. The fear of hypoglycemia in addition to poorly educated nursing staff, insufficient frequency in BG monitoring, failure to recognize changes in insulin requirements and resistance to change, pose a great challenge in most Egyptian hospitals to changing from SSI to weight-based insulin administration and real obstacle to make daily insulin dosing adjustment after first ordering.

\section{Study Limitation}

The main weakness of this study is the small sample size. This could be attributed to two reasons: first, the financial constraint where the study was completely selffunded and all the 70/30 insulin, glargine/glulisine Solostar Flex-Pen ${ }^{\circledR}$ insulin were supplied by the inves- 
tigators; second, the difficulty in recruiting patients with previously set inclusion and exclusion criteria from Cairo University Hospitals. Because treatment in Cairo University hospitals is a completely free service, priority in the provision of case is always given to critical and multiple complicated conditions.

Although nursing errors were associated with major poor patient outcomes in glargine/glulisine group, no appropriate assessment of nursing staff education was conducted during this study.

\section{Conclusions}

With increasing evidence in support of tight glycemic control, new strategies should be implemented to control DM in Egyptian governmental hospitals. With poorly educated nursing staff, basal plus SS insulin failed to provide adequate glycemic control among hospitalized patients. However, it was found that, treatment with tailored 70/30 insulin regimen resulted in both statistically significant glycemic control and shorter length of hospital stay compared to the use of traditional SSI protocol which is inadequate to achieve current recommended glycemic targets in Egyptian patients, when used as the only inpatient treatment for diabetes.

Proper education and training of hospital staff on effective management of patients with diabetes and implementation of standardized subcutaneous scheduled insulin therapy order sets are highly recommended.

\section{Recommendation}

Further trials on larger sample size are recommended together with conducting economical studies comparing the three regimens.

More appropriate assessments of nursing staff quality in both governmental and private hospitals in Egypt are clearly needed.

\section{Ethical Standard}

Informed consent was obtained from all subjects or their surrogate after explanation and understanding of the nature, purpose, and potential risks of the study. The study protocol was approved by the Ethics Committee, Faculty of Pharmacy, Cairo University. This pilot study was conducted according to the Declaration of Helsinki and Good Clinical Practice guidelines [39]. This trial was registered at clinicaltrial.gov with registration identifier (NCT01855243).

\section{Conflict of Interest}

This study was a self-funded, and the authors have no conflict of interest to declare.

\section{Acknowledgements}

The authors acknowledge the efforts made by internal medicine resident and nursing staff, Cairo University teaching hospitals, Egypt.

\section{REFERENCES}

[1] W. H. Herman, M. A. Ali, R. E. Aubert, et al., "Diabetes Mellitus in Egypt: Risk Factors and Prevalence," Diabetic Medicine, Vol. 12, No. 12, 1995, pp. 1126-1131. http://dx.doi.org/10.1111/j.1464-5491.1995.tb00432.x

[2] J. Shaw, R. Sicree and P. Zimmet, "Global Estimates of the Prevalence of Diabetes for 2010 and 2030," Diabetes Research Clinical Practice, Vol. 87, No. 1, 2010, pp. 414. http://dx.doi.org/10.1016/j.diabres.2009.10.007

[3] G. W. Guy, A. V. W. Nunn, L. E. Thomas and J. D. Bell, "Obesity, Diabetes and Longevity in the Gulf: Is there a Gulf Metabolic Syndrome?" International Journal of Diabetes Mellitus, Vol. 1, No. 1, 2009, pp. 43-54. http://dx.doi.org/10.1016/j.ijdm.2009.05.001

[4] H. J. Jiang, D. Stryer, B. Friedman and R. Andrews, "Multiple Hospitalizations for Patients with Diabetes," Diabetes Care, Vol. 26, No. 5, 2003, pp. 1421-1426. http://dx.doi.org/10.2337/diacare.26.5.1421

[5] G. E. Umpierrez, S. D. Isaacs, N. Bazargan, X. You, L. M. Thaler and A. E. Kitabchi, "Hyperglycemia: An Independent Marker of In-Hospital Mortality in Patients with Undiagnosed Diabetes," The Journal of Clinical Endocrinology \& Metabolism, Vol. 87, No. 3, 2002, pp. 978982. http://dx.doi.org/10.1210/jc.87.3.978

[6] J. L. Schnipper, E. E. Barsky, S. Shaykevich, G. Fitzmaurice and M. L. Pendergrass, "Inpatient Management of Diabetes and Hyperglycemia among General Medicine Patients at a Large Teaching Hospital," Journal of Hospital Medicine, Vol. 1, No. 3, 2006, pp. 145-150. http://dx.doi.org/10.1002/jhm.96

[7] S. Clement, S. S. Braithwaite, M. F. Magee, A. Ahmann, E. P. Smith, R. G. Schafer and I. B. Hirsch, "Management of Diabetes and Hyperglycemia in Hospitals," Diabetes Care, Vol. 27, No. 2, 2004, pp. 553-597.

http://dx.doi.org/10.2337/diacare.27.2.553

[8] A. D. Association, "Standards of Medical Care in Diabetes-2013. (Position Statement)," Diabetes Care, Vol. 36, No. 1, 2013, pp. S11-S66.

http://dx.doi.org/10.2337/dc13-S011

[9] G. E. Umpierrez, R. Hellman, M. T. Korytkowski, M. Kosiborod, G. A. Maynard, V. M. Montori, J. J. Seley and G. Van den Berghe, "Management of Hyperglycemia in Hospitalized Patients in Non-Critical Care Setting: An Endocrine Society Clinical Practice Guideline," The Journal of Clinical Endocrinology \& Metabolism, Vol. 97, No. 1, 2012, pp. 16-38. http://dx.doi.org/10.1210/jc.2011-2098

[10] T. Becker, A. Moldoveanu, T. Cukierman and H. C. Gerstein, "Clinical Outcomes Associated with the Use of Subcutaneous Insulin-by-Glucose Sliding Scales to Manage Hyperglycemia in Hospitalized Patients with Pneu- 
monia," Diabetes Research Clinical Practice, Vol. 78, No. 3, 2007, pp. 392-397. http://dx.doi.org/10.1016/i.diabres.2007.05.003

[11] M. F. Magee and S. Clement, "Subcutaneous Insulin Therapy in the Hospital Setting: Issues, Concerns, and Implementation," Endocrine Practice, Vol. 10, No. 1, 2004, pp. 81-88. http://dx.doi.org/10.4158/EP.10.S2.81

[12] B. Boehm, P. Home, C. Behrend, N. Kamp and A. Lindholm, "Premixed Insulin Aspart 30 vs. Premixed Human Insulin 30/70 Twice Daily: A Randomized Trial in Type 1 and Type 2 Diabetic Patients," Diabetic Medicine, Vol. 19, No. 5, 2002, pp. 393-399.

http://dx.doi.org/10.1046/j.1464-5491.2002.00733.x

[13] S. E. Inzucchi, "Management of Hyperglycemia in the Hospital Setting," The New England Journal of Medicine, Vol. 355, No. 18, 2006, pp. 1903-1911. http://dx.doi.org/10.1056/NEJMcp060094

[14] G. E. Umpierrez, D. Smiley, A. Zisman, L. M. Prieto, A. Palacio, M. Ceron, A. Puig and R. Mejia, "Randomized Study of Basal-Bolus Insulin Therapy in the Inpatient Management of Patients with Type 2 Diabetes (RABBIT 2 trial)," Diabetes Care, Vol. 30, No. 9, 2007, pp. 21812186. http://dx.doi.org/10.2337/dc07-0295

[15] G. E. Umpierrez, D. Smiley, S. Jacobs, L. Peng, A. Temponi, P. Mulligan, D. Umpierrez, C. Newton, D. Olson and M. Rizzo, "Randomized Study of Basal-Bolus Insulin Therapy in the Inpatient Management of Ptients with Type 2 Diabetes Undergoing General Surgery (RABBIT 2 Surgery)," Diabetes care, Vol. 34, No. 2, 2011, pp. 256-261. http://dx.doi.org/10.2337/dc10-1407

[16] G. Maynard, D. H. Wesorick, C. O’Malley and S. E. Inzucchi, "Subcutaneous Insulin Order Sets and Protocols: Effective Design and Implementation Strategies," Journal of Hospital Medicine, Vol. 3, No. 5, 2008, pp. 29-41.

[17] G. E. Umpierrez, et al., "Comparison of Inpatient Insulin Regimens with Detemir plus Aspart versus Neutral Protamine Hagedorn plus Regular in Medical Patients with Type 2 Diabetes," The Journal of Clinical Endocrinology \& Metabolism, Vol. 94, No. 2, 2009, pp. 564-569. http://dx.doi.org/10.1210/jc.2008-1441

[18] S. J. Finney, C. Zekveld, A. Elia and T. W. Evans, "Glucose Control and Mortality in Critically Ill Patients," The Journal of the American Medical Association, Vol. 290, No. 15, 2003, pp. 2041-2047.

http://dx.doi.org/10.1001/jama.290.15.2041

[19] I. Stranders, M. Diamant, R. E. van Gelder, H. J. Spruijt, J. W. R. Twisk, R. J. Heine and F. C. Visser, "Admission Blood Glucose Level as Risk Indicator of Death after Myocardial Infarction in Patients with and without Diabetes Mellitus," Archives of Internal Medicine Vol. 164, No. 9, 2004, pp. 982-988.

http://dx.doi.org/10.1001/archinte.164.9.982

[20] J. M. Schoeffler, D. A. K. Rice and D. G Gresham, "70/30 Insulin Algorithm versus Sliding Scale Insulin," The Annals of Pharmacotherapy, Vol. 39, No. 10, 2005, pp. 16061609. http://dx.doi.org/10.1345/aph.1E661

[21] A. Liebl, R. Prager, M. Kaiser, K. Binz and B. Gallwitz, "Biphasic Insulin Aspart 30 (BIAsp30), Insulin Detemir
(IDet) and Insulin Aspart (IAsp) Allow Patients with Type 2 Diabetes to Reach A1C Target: The Prefer study," Diabetes, Vol. 55, Suppl. 1, 2006, p. 123.

[22] D. R. Owens, P. A. Coates, S. D. Luzio, J. P. Tinbergen and R. Kurzhals, "Pharmacokinetics of 125I-Labeled Insulin Glargine (HOE 901) in Healthy Men: Comparison with NPH Insulin and the Influence of Different Subcutaneous Injection Sites," Diabetes Care, Vol. 23, No. 6, 2000, pp. 813-819. http://dx.doi.org/10.2337/diacare.23.6.813

[23] R. Ligthelm, U. Mouritzen, H. Lynggaard, et al., "Biphasic Insulin Aspart Given Thrice Daily Is as Efficacious as a Basal-Bolus Insulin Regimen with Four Daily Injections: A Randomised Open-Label Parallel Group Four Months Comparison in Patients with Type 2 Diabetes," Experimental and Clinical Endocrinology \& Diabetes, Vol. 114, No. 9, 2006, pp. 511-519.

http://dx.doi.org/10.1055/s-2006-924424

[24] M. Clements, J. Tits, B. Kinsley, J. Råstam, H. Friberg and R. Ligthelm, "Improved Glycaemic Control of ThriceDaily Biphasic Insulin Aspart Compared with TwiceDaily Biphasic Human Insulin; a Randomized, OpenLabel Trial in Patients with Type 1 or Type 2 Diabetes," Diabetes, Obesity and Metabolism, Vol. 10, No. 3, 2008, pp. 229-237. http://dx.doi.org/10.1111/j.1463-1326.2006.00687.x

[25] G. Shanmugasundar, A. Bhansali, R. Walia, P. Dutta and V. Upreti, "Comparison of Thrice Daily Biphasic Human Insulin (30/70) versus Basal Detemir \& Bolus Aspart in Patients with Poorly Controlled Type 2 Diabetes Mellitus-A Pilot Study," Indian Journal of Medical Research, Vol. 135, No. 1, 2012, pp. 78-83. http://dx.doi.org/10.4103/0971-5916.93428

[26] R. R. Holman, A. J. Farmer, M. J. Davies, J. C. Levy, J. L. Darbyshire, J. F. Keenan and S. K. Paul, "Three-Year Efficacy of Complex Insulin Regimens in Type 2 Diabetes," The New England Journal of Medicine, Vol. 361, No. 18, 2009, pp. 1736-1747. http://dx.doi.org/10.1056/NEJMoa0905479

[27] S. Yamada, M. Watanabe, O. Funae, Y. Atsumi, R. Suzuki, K. Yajima, Y. Nakamura, T. Kawai, Y. Oikawa and A. Shimada, "Effect of Combination Therapy of a Rapid-Acting Insulin Secretagogue (Glinide) with Premixed Insulin in Type 2 Diabetes Mellitus," Internal Medicine, Vol. 46, No. 23, 2007, pp. 1893-1897. http://dx.doi.org/10.2169/internalmedicine.46.0415

[28] L. M. Dickerson, X. Ye, J. L. Sack and W. J. Hueston, "Glycemic Control in Medical Inpatients with Type 2 Diabetes Mellitus Receiving Sliding Scale Insulin Regimens versus Routine Diabetes Medications: A Multicenter Randomized Controlled Trial," Annals of Family Medicine, Vol. 1, No. 1, 2003, pp. 29-35. http://dx.doi.org/10.1370/afm.2

[29] W. D. Smith, A. G. Winterstein, T. Johns, E. Rosenberg and B. C. Sauer, "Causes of Hyperglycemia and Hypoglycemia in Adult Inpatients," American Journal of HealthSystem Pharmacy, Vol. 62, No. 7, 2005, pp. 714- 719.

[30] G. E. Umpierrez, A. Palacio and D. Smiley, "Sliding Scale Insulin Use: Myth or Insanity?" The American Jo- 
urnal of Medicine, Vol. 120, No. 7, 2007, pp. 563-567. http://dx.doi.org/10.1016/j.amjmed.2006.05.070

[31] I. B. Hirsch, "Sliding Scale Insulin-Time to Stop Sliding," The Journal of the American Medical Association, Vol. 301, No. 2, 2009, pp. 213-214. http://dx.doi.org/10.1001/jama.2008.943

[32] L. A. Browning and P. Dumo, "Sliding-Scale Insulin: An Antiquated Approach to Glycemic Control in Hospitalized Patients," American Journal of Health-System Pharmacy, Vol. 61, No. 15, 2004, pp. 1611-1614.

[33] L. H. Aiken, S. P. Clarke, D. M. Sloane, J. A. Sochalski, R. Busse, H. Clarke, P. Giovannetti, J. Hunt, A. M. Rafferty and J. Shamian, "Nurses' Reports on Hospital Care in Five Countries," Health affairs, Vol. 20, No. 3, 2001, pp. 43-53. http://dx.doi.org/10.1377/hlthaff.20.3.43

[34] A. E. Tourangeau, D. M. Doran, L. M. Hall, L. O'Brien Pallas, D. Pringle, J. V. Tu and L. A. Cranley, "Impact of Hospital Nursing Care on 30 Day Mortality for Acute Medical Patients," Journal of Advanced Nursing, Vol. 57, No. 1, 2007, pp. 32-44. http://dx.doi.org/10.1111/j.1365-2648.2006.04084.x

[35] C. R. Friese, E. T. Lake, L. H. Aiken, J. H. Silber, J.
Sochalski, "Hospital Nurse Practice Environments and Outcomes for Surgical Oncology Patients," Health Services Research, Vol. 43, No. 4, 2008, pp. 1145-1163. http://dx.doi.org/10.1111/j.1475-6773.2007.00825.x

[36] T. A. Lang, M. Hodge, V. Olson, P. S. Romano and R. L. Kravitz, "Nurse-Patient Ratios: A Systematic Review on the Effects of Nurse Staffing on Patient, Nurse Employee, and Hospital Outcomes," The Journal of Nursing Administration, Vol. 34, No. 7-8, 2004, pp. 326-337. http://dx.doi.org/10.1097/00005110-200407000-00005

[37] M. Manojlovich and B. DeCicco, "Healthy Work Environments, Nurse-Physician Communication, and Patients' Outcomes," American Journal of Critical Care, Vol. 16, No. 6, 2007, pp. 536-543.

[38] L. M. Hall, D. Doran, G. R. Baker, G. H. Pink, S. Sidani, L. O'Brien-Pallas and G. J. Donner, "Nurse Staffing Models as Predictors of Patient Outcomes," Medical Care, Vol. 41, No. 9, 2003, pp. 1096-1109. http://dx.doi.org/10.1097/01.MLR.0000084180.07121.2B

[39] World Medical Association, "Declaration of Helsinki," 2013. http://www.wma.net/en/20activities/10ethics/10helsinki/ 